\title{
Female-to-Male Transsexual Animal
}

National Cancer Institute

\section{Source}

National Cancer Institute. Female-to-Male Transsexual Animal. NCI Thesaurus. Code C47918.

An organism that was female at birth based on physical characteristics and has undergone hormonal therapy and/or surgical procedures in order to develop more male physical characteristics. 